\title{
Stimulus averaging and the context for judgment
}

\author{
ALLEN PARDUCCI AND HOWARD THALER, UNIVERSITY OF CALIFORNIA, LOS ANGELES \\ NORMAN H. ANDERSON, UNIVERSITY OF CALIFORNIA, SAN DIEGO
}

Loudness judgments of stimulus sets composed of four bursts of noise were analyzed for two types of contextual effects: between-set and within-set. Experiment 1 demonstrated between-set effects for these four-component stimuli; they were shown to be similar to those found in previous work with single stimuli. Experiment 2 tested an averaging model for within-set contextual effects. The results were inconsistent with the model. One interpretation is that there are within-set effects and that these are caused by shifts in the effective range of stimuli. Altemative interpretations attribute the apparent contextual effects either to an averaging of physical values or to an inappropriate scale of judgment.

Studies of the effects of context upon category judgment ordinarily employ the method of single stimuli in which $S$ categorizes each of a series of stimuli on some dimension of judgment. The series itself usually provides the primary frame of reference, at least with psychophysical judgments. Such judgments can be predicted from the range and relative frequencies of the stimuli in the series (Parducci, 1965).

The traditional method of single stimuli is sometimes modified so that S's task is to form an overall impression. Each overt judgment is applied to a short series or set of single stimuli rather than to the separate components. This suggests a distinction between two types of contextual effects.

First, the sets themselves can be considered as stimulus units. Insofar as the sets function as single stimuli, the frame of reference is established by the different sets in the series. Accordingly, the judgments of the sets should reflect the same contextual effects that have been established for judgments of single stimuli.

A second type of contextual effect may derive from interrelations among the stimuli within each separate set. To make an overall judgment, $s$ must integrate the information in the component stimuli of the set. This integration may be thought of as operating upon implicit judgments of the separate components. But these implicit judgments may themselves be affected by the other components within the same set. If this is so, then the overall judgments should reflect this second source of contextual effects.

However, recent studies of information integration seem to indicate that such within-set effects are negligible (Anderson, 1962a; 1964; 1965). In a typical study, Ss judged the likableness of a person described by a set of personality-trait adjectives. The overall judgment was fairly well described as an average of the values of each separate adjective, independent of the other adjectives in the set. The apparent independence of these verbal materials contrasts markedly with the contextual effects that have been found for judgments of single stimuli, particularly when the stimuli vary on a simple physical dimension. It seemed possible, therefore, that within-set effects might be greater if psychophysical rather than verbal stimuli were used.

Both between-set and within-set contexts are studied here using multi-component, psychophysical stimuli. Experiment 1 manipulates the context between sets. Experiment 2 manipulates the components within the individual sets.

\section{METHOD}

In both experiments, Ss were presented with sets that consisted of four bursts of noise. They were instructed to judge the average loudness of each set "... in comparison with the average loudness of the other sets that you have been presented." They responded by recording numerals, 1 through 6 , to indicate the following category judgments: Very Soft, Soft, Slightly Softer than Average, Slightly Louder than Average, Loud, and Very Loud, respectively.

\section{Stimuli}

The output of a random noise generator was shaped by an electronic switch (50 msec rise-time) and attenuated by one of four alternative resistors to yield intensities of $65,70,75$, or $80 \mathrm{~dB}$ SPL at S's earphones. On each trial, four bursts of noise were presented successively as a set. Each burst lasted $1 \mathrm{sec}$, with a 1-sec interburst interval. A light, presented $1 \mathrm{sec}$ after the fourth burst, was the signal to respond. The total duration of a trial was $12 \mathrm{sec}$.

\section{Experiment 1}

Each set may be characterized by the sum of the dB-values of its four components, and there are 13 such sums. Three groups of 104 sets were selected to yield three distributions of these dB-sums: positively skewed, in which the lower intensities predominate; negatively skewed, in which the higher intensities predominate; and normal. The frequencies are presented at the bottom of Fig. 1.

Each distribution was presented in different quasi- 


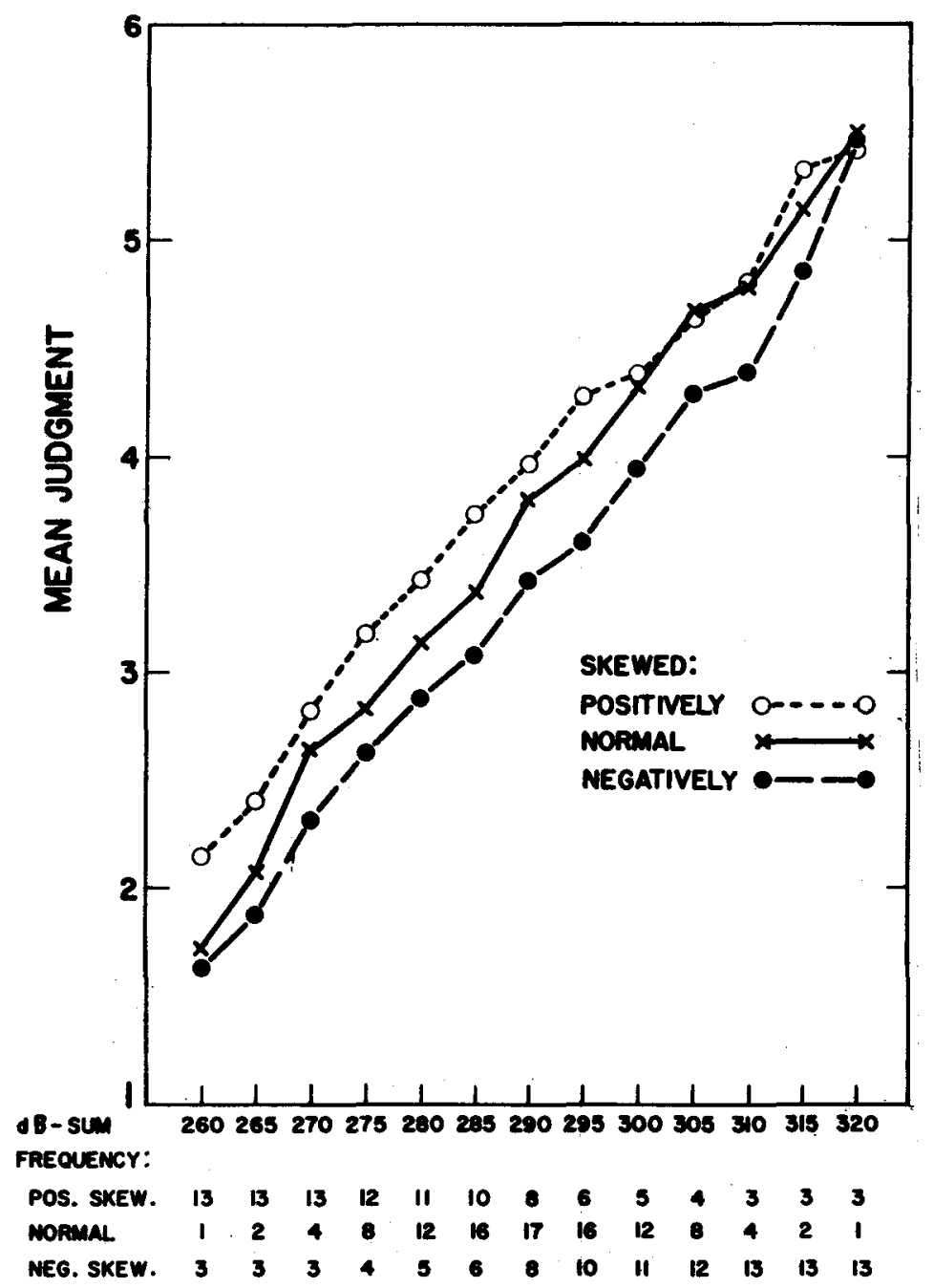

random orders to four squads of four Ss each. The serial order of the four noise intensities within each set was balanced over squads. After 19 warmup sets which had been selected to be representative of each distribution, the 104 regular sets were presented in a gingle session to each squad.

\section{Experiment 2}

At each of the four serial positions in a stimulus set, there are four possible noise intensities. The class of all possible sets thus forms a $4^{4}$ factorial design. All 256 of these permutations were used.

In the initial practice session, 11 college students were presented 64 sets, and the eight Ss with the best loudness discrimination were retained. These eight Ss were presented all 256 sets, 128 on each of the next two days.

Each squad of four Ss received the 256 sets in a different random order. The experimental sessions began with four warmup sets in which the four noise intensities were presented equally often but in random combinations.
Fig. 1. Mean judgments of stimulus sets in the three distributions of Experiment 1. Each set is characterized by the sum of its four $d B$ components as listed on the abscissa.

\section{Experiment 1}

\section{RESULTS ANO DISCUSSION}

Figure 1 shows the mean judgments as a function of the sum of the dB-values of the four noise bursts in the sets. Although some of the end points are based on very few responses, the general trend is clear. A given set receives a higher judgment in the positively-skewed distribution, in which sets of lower intensities predominate, than in the negatively-skewed distribution in which sets of higher intensities predominate. This difference is quite rellable. Consider, for example, the middle stimulus set, with a dB-sum of 290. Only 1 of the $16 \mathrm{Ss}$ in the negatively-skewed condition judged this set as high as the mean for the positively-skewed condition.

Taken as a whole, the three functions in Fig. 1 resemble those obtained previously for judgments of the size of squares presented as single stimuli (Parducci, 1965; Figs. 5 and 6). The steeper slope for the normal distribution is also consistent with the previous predictions for similar distributions. The main implications of Experiment 1 are thus easily sum- 
marized. If multi-component stimuli are treated as units, judgments of them appear to follow the same laws as judgments of single stimuli at both the empirical and theoretical level.

\section{Experiment 2}

The objective of this experiment was to test for within-set contextual effects, that is, for interaction among the components of a set. Since the judgment was made to the set as a whole, these tests are somewhat indirect and inferential. The serial position effects and the two-way data tables will be given first. Alternative interpretations of the latter will then be considered.

Serial position. Figure 2 presents the mean judgments as a function of serial position and stimulus intensity. Each point is an average over 64 sets and elght Ss. Since the serial posittons, which are plotted on the abscissa, correspond to the factors of the $4^{4}$ design, the four data points at each abscissa value correspond to the main effects of the noise intensities at each serial position.

These data exhibit a strong recency effect. The top curve represents sets that had at least one $80-\mathrm{dB}$ burst; its upward slope shows that the set as a whole is judged louder when this loudest burst is closer to the end of the set. Similariy, the bottom curve represents sets that contained one or more 65-dB bursts; its downward slope shows that such sets are judged softer when this softest burst is closer to the end.

Inspection of the corresponding single-subject graphs showed considerable individual differences in amount of recency. For two Ss, the serial curves were essen-

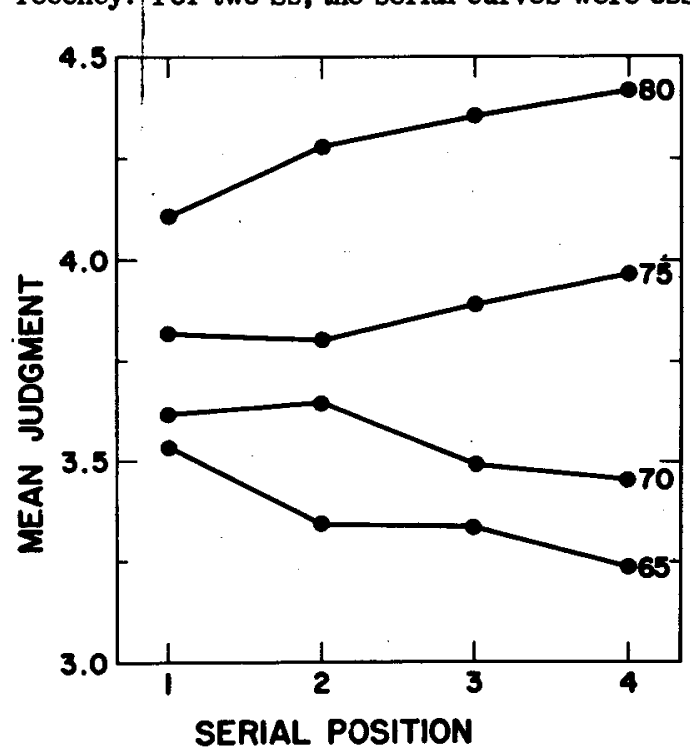

Fig. 2. Serial position curves, Experiment 2. Each data point is averaged over all sets containing the specified dB value at the given serial position. tially horizontal, indicating little or no differential effect of serial position. However, separate statistical analyses for each $\mathbf{S}$ showed that each serial position had a significant influence on the judgments in 31 of 32 instances (8 Ss $\times 4$ positions), the single exception being the first position for one $S$.

The relative spread of the curves is a surprising feature of Fig. 2. The differences in average judgment increase with stimulus intensity. Thus, the average difference between the 75- and $80-\mathrm{dB}$ curves is approximately 2-1/2 times the average difference between the 65- and 70-dB curves. Unequal spacing among the curves is noteworthy since loudness judgments tend to be linearly related to $\mathrm{dB}$ for single stimuli in this range (e.g., Galanter \& Messick, 1961). If it were not for the predominance of "louder" judgments, this nonlinearity would suggest an endeffect or cat-off at the bottom of the scale.

Two-way data tables. "Each panel of Fig. 3 plots loudness judgments for one of the six pairs of serial positions. In each panel, the later of the two positions is represented on the abscissa, and the intensities at the earlier position correspond to the four curves. In the upper-left panel, for example, the abscissa values are the four intensities in Position 2, and the four curves correspond to the four intensities in Position 1. Each data point represents the mean judgment of eight Ss for each of 16 sets, being averaged over all intensities in the other two serial positions.

The upward trend of each curve reflects the increasing stimulus intensity at the later of the two relevant serial positions. The differences among the curves within each panel reflect the effect of stimulus intensity in the earlier serial position. In each of the three upper panels, for example, the separation of the curves reflects the differential effects of the intensities in Position 1 on the final response. There is the same tendency for the relative separation to be greater at the higher intensities that was noted for the serial position curves in Fig. 2.

To establish the reliability of the main effects of serial position noted above, a separate analysis of variance was first run for each $S$ with the fourway interaction used as the error term. Significant interactions showed a chance pattern, except for one $S$. An overall analysis was run on the pooled data. Each systematic source, including the fourway interaction, was then tested against its interaction with Ss. An auxdliary analysis that omitted the exceptional $S$ just mentioned gave basically the same pattern of results as the overall analysis.

The main statistical analyses are most conveniently summarized in connection with the two-way tables, each of which is represented by one of the panels of Fig. 3. Listed in each panel are the Mean Squares for that interaction and for its error term ( $d f=9$ and 


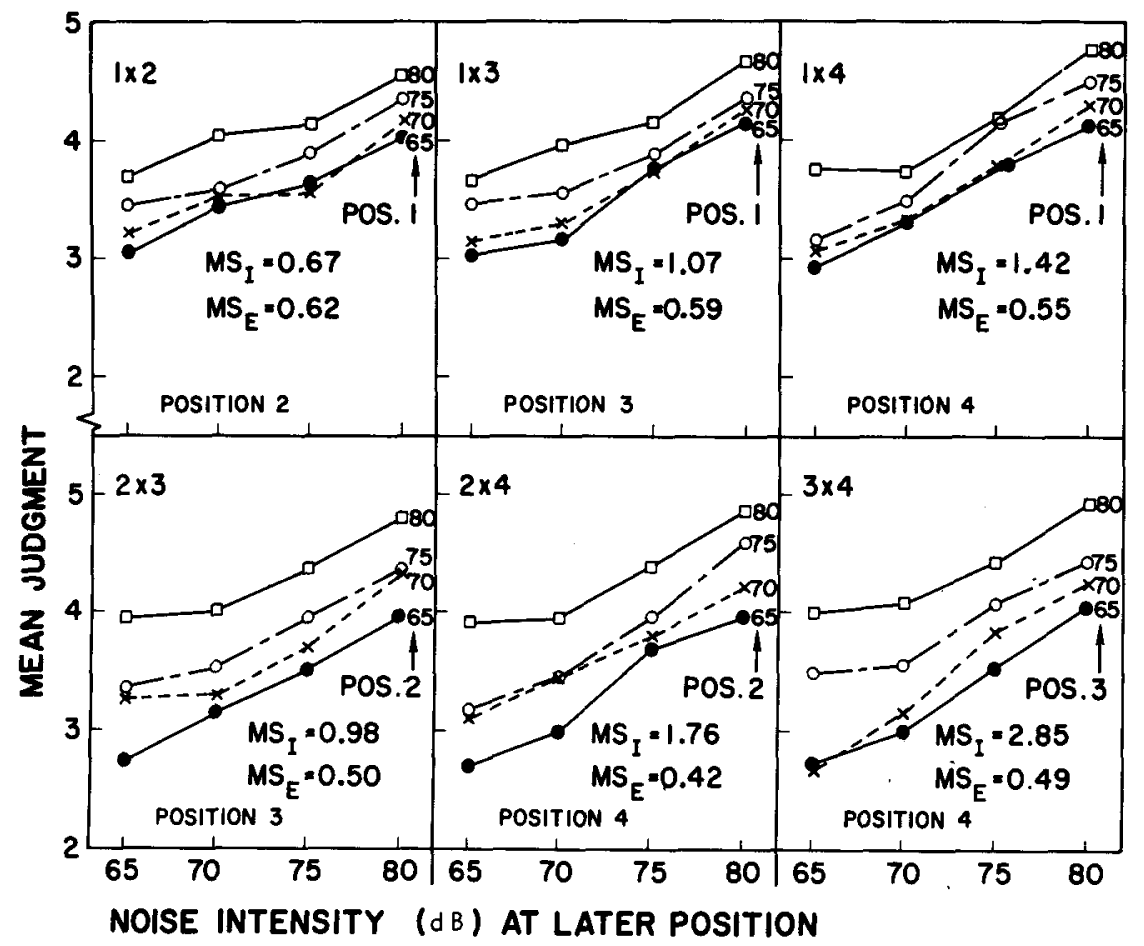

Fig. 3. Two-way data tables for each pair of serial positions, Experiment 2. Each data point is averaged over the 16 combinations of intensities at the other two serial positions.
63, respectively). The critical value of $F$ at the .05 level is 2.03 so that each of the three interactions involving Position 4 is significant.

Geometrically, the null hypothesis tested by each two-way interaction is that the curves of the corresponding panel are parallel. Significant interaction thus implies a reliable degree of nonparallelism. Inspection of Fig. 3 suggests that the main source of nonparallelism is a convergence to the right. A quick check was obtained by calculating a double differencescore as follows: the difference between the $80-\mathrm{dB}$ and $65-\mathrm{dB}$ curves at the leftmost, 65- $\mathrm{dB}$ abscissa value, minus the difference between the same curves at the rightmost, $80-\mathrm{dB}$ abscissa value. This specific comparison was tested against the listed error mean square for each panel, and was significant in the three lower panels $(F=7.57,7.79$, and 11.23 , respectively; $d f=1 / 63$ ).

In addition to the three two-way interactions, the two three-way interactions that involved Positions 3 and 4 and the four-way interaction were significant with $F$ ratios of nearly 1.70 in each case. In contrast to the two-way interactions, graphical analyses failed to reveal a consistent overall pattern in these higherorder interactions. The data did suggest, however, that the effect of intensity in any one position varied inversely with the intensities at the other positions.

Averaging model. This model, as applied to serial information integration, assumes that the response to any set is a sum or mean of the effective values of the components of the set, suitably weighted for serial position. Thus, each response can be written as $\Sigma \mathbf{s}_{\mathbf{k}} \mathbf{w}_{\mathbf{k}}$, where $\mathbf{s}_{\mathbf{k}}$ is the effective loudness of the burst in Position $k$, and $w_{k}$ is the weight associated with Position k. The model assumes that the effective value of any intensity is the same in all sets, and similarly that the weight associated with Position $k$ is the same in all sets.

This model allows the use of ordinary analysis of variance to estimate parameters and to test goodness of fit (Anderson, 1962a; 1964). The correlation between the observed means and those predicted using the parameters estimated by the model was .914. Although this correlation is moderately large, it has shortcomings even as an index of the degree of fit between model and data. The proper test of the model is made on the discrepancies from prediction. This test is straightforward since the model implies that all interactions are zero. In particular, therefore, the curves of each panel of Fig. 3 should be parallel up to sampling fluctuation. As has been seen, however, three of the panels exhibit significant nonparallelism, and three of the higher-order interactions were also significant. The data thus appear to be inconsistent with the averaging model.

These discrepancies may reside in the response scale instead of the model. The averaging model requires a valid numerical response, and distortion in the scale of overt judgment could produce statistical interaction even if $S$ is internal impression followed the model. Scale distortion might have arisen from scale end-effects as well as from the use of only six response categories. The work on information integration cited earlier has used rating scales with 

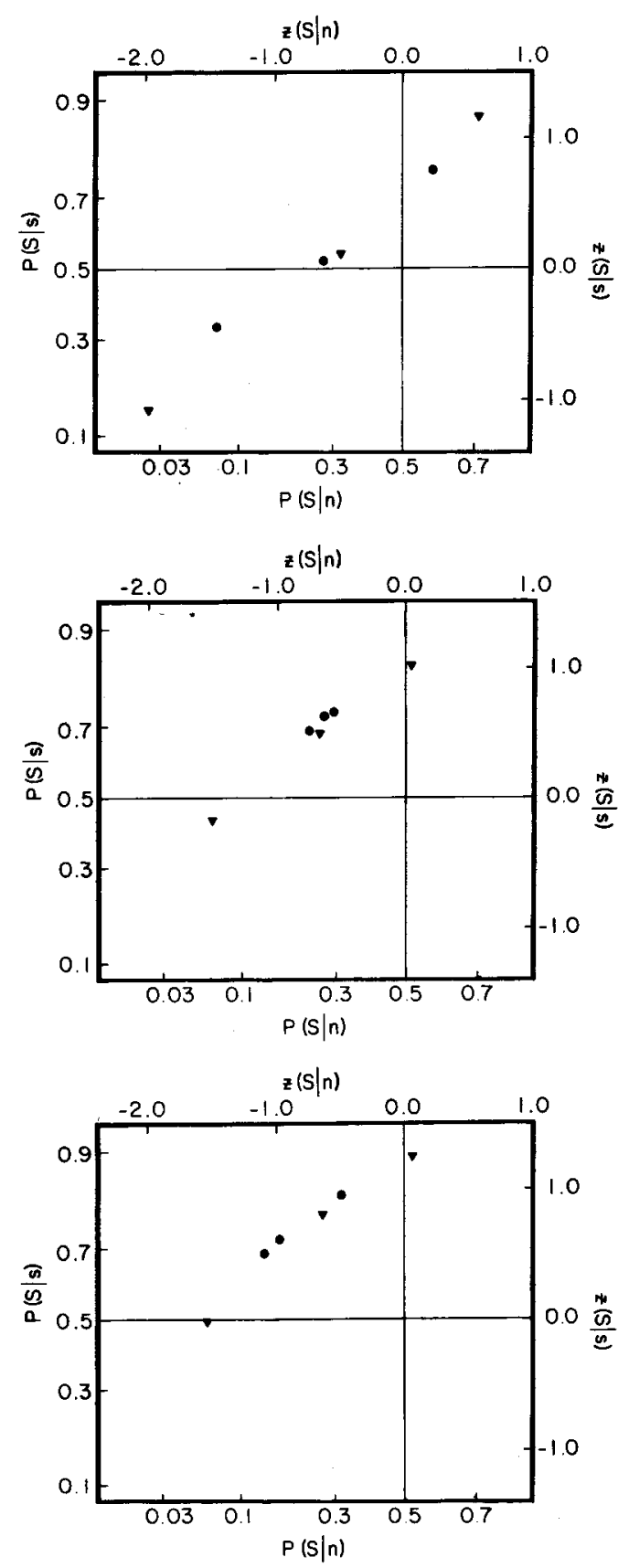

Fig. 3. Proportion of hits plotted against proportion of false alarms on double Gaussian coordinates. Experiment 3: rating-scale method, $\nabla$; binary method, - . Top: Observer WC; luminance of line, 0.85 mL: Middle: Observer SA; luminance of line, $1.08 \mathrm{~mL}$; Bottom: Observer SA; luminance of line, $1.08 \mathrm{~mL}$. After every block of trials, observer was told the difference between the numbers of correct and incorrect responses.

these points are ordered in the same way, namely, the point representing the lowest hit and false alarm rates coming from blocks of trials where $P_{S}=0.25$, while the one representing the highest rates coming from blocks where $P_{S}=0.75$. A similar effect of presentation probability on response criterion has been obtained with the binary procedure whenever the $O$ is given trial-to-trial feedback (Tanner, Haller, \& Atkinson, 1967).

The preceding discussion of the effects of presentation probability apply equally well to the results of Experiment 1 (Fig. 1) and of Experiment 2 (Fig. 2). Apparently it makes little difference whether one or three different presentation probabilities are used within an experimental session.

Figure 3 (top to bottom) consists of plots of the results of Experiment 3 , where the rating method was used in conjunction with $P_{S}=0.5$, and the binary procedure with $\mathrm{P}_{\mathrm{S}}=0.25,0.50$, and 0.75 . Here again, all the data points in each panel can be fit by a single ROC curve which is a straight line of slope less than unity. The one consistent difference between the circles (binary procedure) and the triangles (rating procedure) is that the former are closer to each other than are the latter. The relative ineffectiveness of changing presentation probability as a means of shifting response criterion with the binary procedure is particularly noteworthy in Fig. 3 (middle), where the false alarm rates range from 0.23 for $P_{S}=0.25$ to 0.28 for $P_{S}=0.75$. As Fig. 3 (bottom) shows, this same $O$ did produce a somewhat wider range of false alarm rates when, in addition to receiving feedback after every trial, he was also told his score (number of correct responses minus number of incorrect responses) after every block of trials.

\section{CONCLUSIONS}

According to the results of the present experiments, an observer's performance in a simple visual detection task can be described by a single ROC curve regardless of the presentation probability of the signal and of the number of criteria he must simultaneously maintain. However, it must be stressed that these experiments covered a narrow range of presentation probabilities $(0.25$ to 0.75$)$, number of simultaneous criteria ( 1 to 3 ), and detectability of signals $\left(d^{\prime}=\mathbf{0} .6\right.$ to 2.0). Furthermore, because of the permissive nature of the instructions to observers, the median range of criterion values adoped by any observer in any experiment was only $2.2 \sigma_{n}$ units. It remains to be seen whether the above generalization about the equivalence of ROC curves will hold over a wider range of relevant experimental conditions.

\section{References}

Egan, J. P., Schulman, A. I., \& Greenberg. G. Z. Operating characteristics determined by binary decisions and by ratings, $J$. Acoust. Soc. Amer., 1959, 31, 768-773.

Green, D. M., \& Swets, J. A. Signal detection theory and psychophysics. New York: Wiley, 1966. 

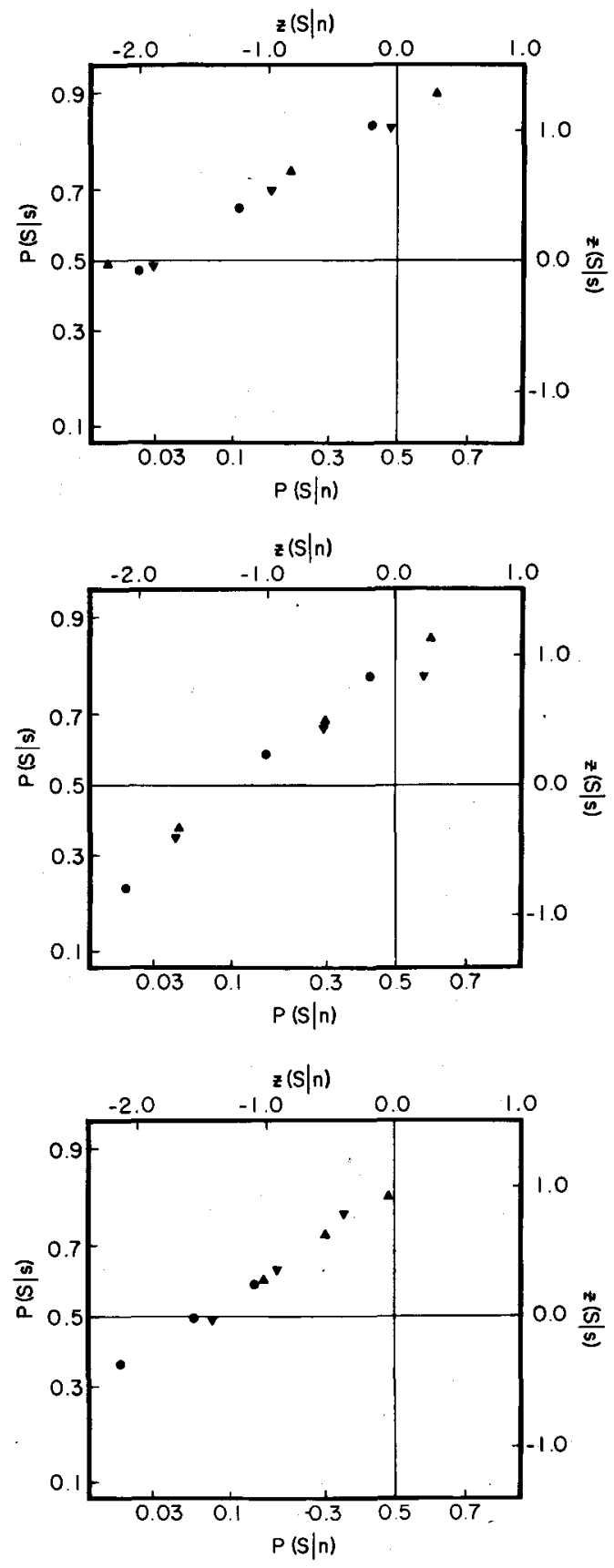

Fig. 1. Proportion of hits plotted against proportion of false alarms on double Gaussian coordinates. Experiment 1: rating-scale method. Luminance of line, $1.08 \mathrm{~mL}$. Data gathered with $P_{S}=0.25$, 0.50 , and 0.75 are represented by $\odot, \Delta$, and $\nabla$ respectively. Top: Observer WC; Middle: Observer SA; Bottom: Observer SH.

from one $O$ in one experiment. A single straight line of slope less than unity would appear to provide a good visual fit to each set of data. ${ }^{2}$ of course, separate lines can be used to fit the data points based on different presentation probabilities. However, the differences between the three lines for each figure would be rather small, and would not be consistent from figure to figure. Therefore, these results offer no grounds for doubting that the rating-scale method generates segments of the same ROC curve, regardless of the presentation probability of the signal.

All previously reported ROC curves from visual detection experiments have also been linear on double Gaussian coordinates, and have also had slopes less than unity (Swets, Tanner, \& Birdsall, 1961; Nachmias and Steinman, 1963; Wickelgren , 1967). Such ROC curves could arise if $f_{s}(x)$ and $f_{n}(x)$, the distributions of internal events on which detection is based, are both Gaussian, with the standard deviation of $f_{\mathrm{S}}(\mathrm{x})$ being larger than that of $f_{n}(x)$.

Figures 1 and 2 show that changing the presentation probability of the signal had one very marked and systematic effect: It altered each of the O's three response criteria. In general, the smaller the presentation probability, the lower each criterion was set. This can be seen by considering separately the points occupying the same ordinal position within each set. of three points obtained with the same presentation probability in each figure. In 16 out of 18 cases
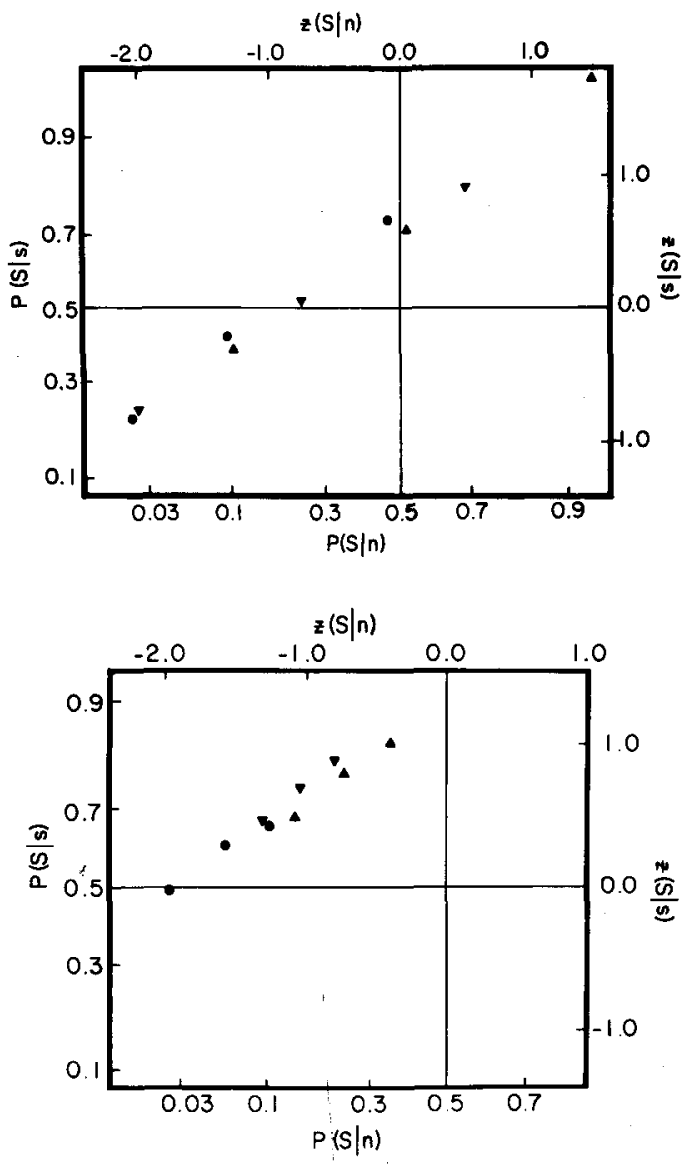

Fig. 2. Proportion of hits plotted against proportion of false alarms on double Guassian coordinates. Experiment 2: rating-scale method. Data gathered with $P_{s}=0.25,0.50$, and 0.75 are represented by $\boldsymbol{D}, \Delta$, and $\nabla$ respectively. Top: Observer WC; luminance of line, 0.85 mL; Bottom: Observer SH; luminance of line, $1.08 \mathrm{~mL}$. 
Markowitz, J., \& Swets, J. A. Factors affecting the slope of empirical ROC curves: comparison of binary and rating responses. Percept. \& Psychophys., 1967, 2, 91-101.

Nachmias, J. Effect of exposure duration on visual contrast sensitivity with square wave gratings. J. Opt. Soc. Amer., 1967, 57, 421-427.

Nachmias, J., \& Steinman, R. M. Study of absolute visual detection by the rating-scale method. J. Opt. Soc. Amer., 1963, 53, 12061213

Swets, J. A., Tanner, W. P., Jr. \& Birdsall, T. G. Decision processes in perception. Psychol. Rev., 1961, 68, 301-340.

Tanner, W. P., Jr., Haller, R. W., \& Atkinson, R. C. Signal recognition as influenced by presentation schedules. Percept. \& Psychophys., 1967, 2, 349-359.
Watson, C. S., Rilling, M. E.; \& Bourbon, W. T. Receiver-operating characteristics determined by a mechanical analog to the rating scale. J. Acoust. Soc. Amer., 1964, 36, 283-288.

Wickelgren, W. A. Strength theories of dis junctive visual detection. Percept. \& Psychophys., 1967, 2, 331-338.

\section{Notes}

1. This work was supported by grant NB06050 from the National Institute of Neurological Diseases and Blindness. USPHS. The assistance of Mr. George Kibler is gratefully acknowledged.

2. Unfortunately, no valid statistical techniques are available for fitting such data.

(Accepted for publication October 15, 1967.) 